\title{
Antimoniato de meglumina
}

No ano de 2000, o Ministério da Saúde, por meio de processo licitatório, adquiriu o antimoniato de $\mathrm{N}$-metil glucamina, cujo nome comercial é o antimoniato de meglumina do laboratório Eurofarma (produto registrado como similar ${ }^{-1}$ na Agência Nacional de Vigilância Sanitária/ Ministério da Saúde) para o tratamento dos pacientes portadores de leishmanioses. Esse medicamento foi distribuído diretamente do produtor para as Secretarias Estaduais de Saúde e Coordenações Regionais da Fundação Nacional de Saúde (FUNASA), a partir de 30 de abril próximo passado.

$\mathrm{O}$ antimoniato de $\mathrm{N}$-metil glucamina é o fármaco de primeira escolha para o tratamento das leishmanioses. Este fármaco é um produto que requer administração cautelosa, sob acompanhamento clínico e laboratorial, por ser cardiotóxico, hepatotóxico e nefrotóxico.

A partir de outubro de 2000 a Fundação Nacional de Saúde/FUNASA, por meio do Centro Nacional de Epidemiologia, recebeu notificações de eventos adversos de severidade grave (abscessos estéreis, intensas dores musculares e nas articulações reações alérgicas) em pacientes que encontravam-se fazendo uso do referido medicamento. Essas notificações iniciaram-se nos Estados do Piauí, Paraná e Mato Grosso, estendendo posteriormente a outras unidades federadas.

O Centro Nacional de Epidemiologia (CENEPI), em 10 de outubro de 2000 convocou o Comitê Técnico Assessor em Diagnóstico e Tratamento das Leishmanioses, que reúne os principais especialistas brasileiros, para analisar esses relatos, tendo o mesmo recomendado a suspensão da utilização dos lotes desse medicamento relacionados com os eventos notificados. O CENEPI/FUNASA, nessa ocasião, informou esse fato ao Ministério da Saúde e a Agência Nacional de Vigilância Sanitária e enviou ofícios aos Secretários Estaduais e Coordenadores Regionais da FUNASA suspendendo a utilização dos lotes 05/00A, 05/00B, 10/00A, 10/00B, 16/00, 17/00A E 23/00B.

O CENEPI/FUNASA iniciou uma investigação epidemiológica mais detalhada das reações adversas notificadas nos Estados do Piauí e Paraná. No Piauí, a investigação abrangeu 99 pacientes de Teresina, correspondendo a $70 \%$ do total dos submetidos ao tratamento com esse medicamento. Nesse estudo, foi encontrada uma taxa de ocorrência de enduração ou abscessos extensos no local da aplicação do medicamento de $38,4 \%$ e $8 \%$ dos pacientes, respectivamente, apesar desses tipos de reação adversa não serem esperados de ocorrer nessas proporções. Dentre os lotes usados, o 05/00A, o 05/00B e o 10/00B apresentaram elevadas taxas de ataque para enduração ou abscessos de 43,5\%, $22,2 \%$ e $33,3 \%$, respectivamente. Para o lote $17 / 00 \mathrm{~A}$, a taxa de ataque foi de $5,5 \%$ e os três pacientes que fizeram uso do lote 09/00 B não apresentaram reações.

Realizou-se também um estudo epidemiológico que comparou os pacientes que utilizaram esses lotes com um grupo de pacientes que utilizou outros lotes do mesmo medicamento, encontrando-se associação estatística com o desenvolvimento de enduração e/ou abscessos, verificando-se Riscos Relativos de 2,6 ( IC de 1,7 a 3,8), 2,5 (IC de 1,6 a 3,7) e 2,3 (IC de 1, 5 a 3,6), respectivamente, para os lotes 05/00 A, 05/00B 10/00B. A análise desse estudo não encontrou associação entre os eventos e a dose aplicada, o sexo e a idade dos pacientes. Os estudos no Estado do Paraná encontram-se em fase de análise dos resultados.

O CENEPI/FUNASA providenciou o encaminhamento de amostras desses e de outros lotes relacionados com a ocorrência dos eventos adversos para a realização de análises laboratoriais. Como o referido medicamento não possui informação científica publicada nas farmacopéias brasileira e internacional, que estabeleçam parâmetros para o controle de qualidade, utilizou-se a comparação com o medicamento registrado no Ministério da Saúde como inovador ${ }^{2}$ (Glucantime-Aventis).

Foram encaminhadas ao Instituto Oswaldo Cruz/ FIOCRUZ amostras de quatro lotes relacionados com eventos adversos nos Estados do Piauí, Rio de Janeiro e Paraná, o 23/00B, o 10/00B, o 05/00B, o 17/00 A, do produto da Eurofarma e do lote 266 do laboratório Aventis, para a realização de microscopia eletrônica. 0 resultado desta microscopia, pelas técnicas de varredura e transmissão, detectou a presença de estruturas fibrosas em todos os lotes do antimoniato de meglumina da Eurofarma analisados. No lote analisado do medicamento inovador do laboratório Aventis, não foram detectadas estruturas fibrosas. O Instituto Oswaldo Cruz sugeriu que fosse realizada a pesquisa de metais para esclarecer esse achado.

Por não existirem referências de Limite Máximo Esperado (LME) de metais pesados para este medicamento, pesquisou-se na farmacopéia brasileira ( $3^{\mathrm{a}}$ edição) os LME para outros medicamentos injetáveis. Os valores encontrados como LME para esses medicamentos tiveram uma variação de 10 a $25 \mathrm{mg} / \mathrm{l}$.

1, 2. Produto similar é todo produto registrado no Ministério da Saúde a partir de um produto com as mesmas características e princípio ativo já registrado, que por sua vez é denominado inovador.

Endereço para correspondência: CENEPI/FUNASA. SAS, Quadra 4, Bloco N, Sala 707, 70058-902 Brasília, DF, Brasil.

Tel: 5561 314-6332

Recebido para publicação em 20/02/01 
O CENEPI encaminhou amostras dos lotes 05/00B, 10/00B, 17/00 A e 23/00B do medicamento produzido pela Eurofarma e do lote 266 do produto da Aventis ao Laboratório de Análises Químicas Industriais e Ambientais da Universidade Federal de Santa Maria para análise de metais pesados. O laboratório foi escolhido por ser o responsável pelos estudos que estão estabelecendo os parâmetros de referência para esse medicamento. Os resultados detectaram, em todos os lotes produzidos pela Eurofarma, a presença de arsênio, com valores variando de $44,45 \mathrm{mg} / \mathrm{l}$ a $84,60 \mathrm{mg} / \mathrm{l}$ e de chumbo, variando de $24,29 \mathrm{mg} / \mathrm{l}$ a $52,31 \mathrm{mg} / \mathrm{l}$. No lote do produto da Aventis, os valores encontrados foram de $0,26 \mathrm{mg} / \mathrm{l}$ para o arsênio e menor que $0,20 \mathrm{mg} / \mathrm{l}$ para o chumbo.

Apresentamos a seguir o quadro comparativo dos quantitativos de arsênio, chumbo e o somatório de todos os metais pesados detectados nos lotes 23/00B, 10/00 A (Eurofarma) e 266 (Aventis), com os respectivos valores de referência, considerando-se como exemplo um paciente de $60 \mathrm{~kg}$.

\begin{tabular}{|c|c|c|c|c|c|c|c|}
\hline \multirow{2}{*}{$\begin{array}{l}\text { Metal } \\
\text { pesado }\end{array}$} & \multirow{2}{*}{$\begin{array}{c}\text { Valor de } \\
\text { Referência (VR) } \\
\text { mg/kg/dia }\end{array}$} & \multicolumn{2}{|c|}{$\begin{array}{l}\text { Lote } 23 / 00 B \\
\text { Eurofarma }\end{array}$} & \multicolumn{2}{|c|}{$\begin{array}{c}\text { Lote 10/00A } \\
\text { Eurofarma }\end{array}$} & \multicolumn{2}{|c|}{$\begin{array}{c}\text { Lote } 266 \\
\text { Aventis }\end{array}$} \\
\hline & & $\begin{array}{c}\text { resultado } \\
\mathrm{mg} / \mathrm{l}\end{array}$ & $\begin{array}{c}\text { dose recebida* }^{*} \\
\mathrm{mg} / \mathrm{kg} / \mathrm{dia}\end{array}$ & $\begin{array}{c}\text { resultado } \\
\mathrm{mg} / \mathrm{l}\end{array}$ & $\begin{array}{c}\text { dose recebida* } \\
\mathrm{mg} / \mathrm{kg} / \mathrm{dia}\end{array}$ & $\begin{array}{c}\text { resultado } \\
\mathrm{mg} / \mathrm{l}\end{array}$ & $\begin{array}{c}\text { dose recebida* } \\
\mathrm{mg} / \mathrm{kg} / \mathrm{dia}\end{array}$ \\
\hline \multirow[t]{2}{*}{ Arsênio } & oral $0,0003^{\star \star}$ & $84,60 \#$ & 0,0197 & 46,31 & 0,0108 & 0,26 & 0,00006 \\
\hline & & & $\begin{array}{c}65,8> \\
\text { que o VR }\end{array}$ & & $\begin{array}{c}36> \\
\text { que VR }\end{array}$ & & $<$ que o VR \\
\hline \multirow[t]{2}{*}{ Chumbo } & oral $0,007^{\star \star \star}$ & 42,57 & 0,0099 & 52,31\#\# & 0,0122 & 0,20 & 0,00005 \\
\hline & & & $\begin{array}{c}1,4> \\
\text { que o VR }\end{array}$ & & $\begin{array}{c}1,7> \\
\text { que o VR }\end{array}$ & & $<$ que o VR \\
\hline \multirow{3}{*}{$\begin{array}{l}\text { Total de } \\
\text { metais } \\
\text { pesados }\end{array}$} & 10 a $25 \mathrm{mg} / /^{\star \star \star \star}$ & 138,41 & - & 111,39 & - & 2,40 & - \\
\hline & & $5,5>$ que & & $4,4>$ que & & $10>$ que & \\
\hline & & o maior VR & & o maior VR & & o maior VR & \\
\hline \multicolumn{8}{|c|}{$\begin{array}{l}{ }^{*} \text { Cálculo estimado para um paciente de } 60 \mathrm{~kg} \text {, com dosagem de } 20 \mathrm{mg} / \mathrm{SbV} / \mathrm{kg} / \mathrm{dia}(14 \mathrm{ml} / \mathrm{dia} \text {, por via parenteral, conforme a dosagen } \\
\text { preconizada para tratamento das leishmanioses. }\end{array}$} \\
\hline \multicolumn{8}{|c|}{$\begin{array}{l}\text { ** Nível para risco mínimo - Referência da Agency for Toxic Substances and Disease Registry - Centers for Disease Prevention an } \\
\text { Control/CDC }\end{array}$} \\
\hline \multicolumn{8}{|c|}{ 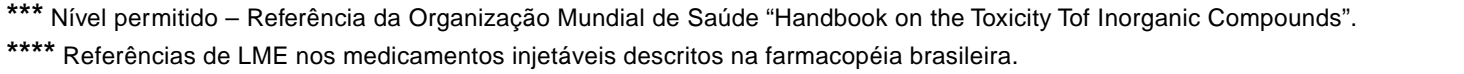 } \\
\hline
\end{tabular}

Nos dados acima verifica-se que o antimoniato de meglumina da Eurofarma apresenta uma quantidade de arsênio que varia de 36 a 65 vezes maior que o valor de referência para adminitração por via oral. No lote 266 produzido pela Aventis os valores encontrados, $0,00005 \mathrm{mg} / \mathrm{kg} / \mathrm{dia}$, estão abaixo da referência. Com relação ao chumbo, os lotes 23/00B e 10/00A apresentam resultados de 1,4 e 1,7 vezes maior que o valor de referência, por via oral, respectivamente.

Quanto ao somatório dos resultados dos metais pesados, os lotes da Eurofarma apresentaram 4,4 e 5,5 vezes mais metais que o maior valor de referência. No lote 266 da Aventis o valor encontrado foi 10 vezes menor que o maior valor de referência.

Os valores de referência estabelecidos para o arsênio e o chumbo estão relacionados com a administração por via oral. Como o antimoniato de meglumina é uma droga injetável, torna-se ainda mais importante que os valores observados encontrem-se abaixo dos valores de referência, sob pena da presença dessas substâncias poderem trazer riscos à saúde dos pacientes portadores de leishmaniose visceral e tegumentar americana tratados com essa medicação.

A partir da notificação dos eventos adversos, a Coordenação de Acompanhamento Tecnológico Farmacêutico do Ministério da Saúde, encaminhou amostras dos lotes 05/00B, 10/00B e 17/00A do produto da Eurofarma, para realização de análise físico-química. Os resultados dos exames reprovaram os lotes 10/00A, 10/00B, por apresentarem $\mathrm{pH}$ abaixo do valor de referência. Os outros dois lotes foram aprovados nesse exame.

O Instituto Nacional de Controle de Qualidade em Saúde (INCQS)/FIOCRUZ também vem realizando análises similares no referido medicamento, com resultados que evidenciam níveis elevados de arsênio e chumbo.

Em conclusão, o antimoniato de meglumina da Eurofarma contém níveis elevados de arsênio e chumbo, 
quando comparados aos valores de referência para a administração por via oral e ao medicamento inovador. Estes metais, em altas dosagens podem causar efeitos graves no organismo humano tais como diminuição de leucócitos e hemácias no sangue, efeitos aditivos nos tecidos respiratórios e sistema nervoso central, alterações severas no ritmo cardíaco, aumentar o risco para o câncer, entre outros.

Com base nas evidências científicas já estabelecidas pelo estudo epidemiológico e pelas análises realizadas, recomendamos:

- Suspensão do uso de todos os lotes do antimoniato de meglumina da Eurofarma que se encontra disponível na rede de saúde, como medida preventiva da ocorrência de reações adversas graves e da exposição a altos níveis de arsênio e chumbo, exceto em casos de calazar que impliquem risco de vida para os pacientes. Nesses casos, o monitoramento de possíveis reações adversas deve ser intensificado.

A FUNASA vem adotando as seguintes condutas, conjuntamente aos demais órgãos do Ministério da Saúde:

- realização de exames minuciosos em todos os lotes do referido medicamento, com os devidos procedimentos fiscais, por parte da Agência Nacional de Vigilância Sanitária.

- Aquisição imediata de substituto do referido medicamento para evitar a descontinuidade no tratamento dos pacientes de leishmanioses.

Brasília, 2 de fevereiro de 2001

Jarbas Barbosa da Silva Junior

Diretor do CENEPI 